\title{
APPLICATION OF AN XML-BASED DATABASE FOR PROBABILISTIC ANALYSIS
}

\author{
Harry Millwater ${ }^{\square}$ \\ The University of Texas at San Antonio \\ San Antonio, Texas \\ Simeon H.K. Fitch ${ }^{\dagger}$ \\ Mustard Seed Software \\ San Antonio, Texas \\ Michael P. Enright and Luc Huyse \\ Southwest Research Institute \\ San Antonio, Texas
}

\begin{abstract}
$\underline{\text { Abstract }}$
The Extensible Markup Language, XML, is used as database file format for a probabilistic fracture mechanics program, DARWIN ${ }^{\mathrm{TM}}$, which is used for risk assessment of commercial aircraft engine rotors. The database is used for storage and retrieval of probabilistic results during computations, for transferring data from the computational code to the graphical user interface, generating HTML output reports, and for archiving the results. The advantages of using an XML database file format are discussed through an application example.
\end{abstract}

\section{Introduction}

The XML specification has emerged within the last few years as a set of rules from the World Wide Web Consortium (W3C) as a means for designing text formats for structuring data [1]. XML defines a text markup language using elements (words bracketed by ' $<$ ' and ' $>$ ') and attributes (of the form name="value") to describe the data. By combining elements, attributes and the data itself in a file, the data and a description of the data are self-contained.

There are a number of appealing features of XML that may be useful, see Table 1. Here we implement XML as the underlying database file format for a probabilistic fracture mechanics code DARWIND [2]. The primary purpose of this database is to store and retrieve information during computations and to transfer

\footnotetext{
$\square$ Assistant Professor, Member AIAA

${ }^{\dagger}$ Consultant

${ }^{*}$ Senior Research Engineer, Research Engineer
}

information from the computational code to the graphical user interface.

\section{Table 1 XML Features}

\begin{tabular}{|l|}
\hline $\begin{array}{l}\text { Formally standardized by World Wide Web } \\
\text { Consortium (W3C) }\end{array}$ \\
\hline License free \& non-proprietary \\
\hline Platform-independent \\
\hline Well-developed and available tools \\
\hline $\begin{array}{l}\text { Easily transformed to other formats with the Extensible } \\
\text { Stylesheet Language (XSL) }\end{array}$ \\
\hline $\begin{array}{l}\text { Easily validated via Document Type Definition (DTD) } \\
\text { or XML Schema files. }\end{array}$ \\
Probabilistic Fracture Mechanics Application \\
\hline Example
\end{tabular}

The probabilistically-based damage tolerance software code DARWING (Design Assessment of Reliability With INspection) has been described elsewhere and is only briefly outlined here [2-4]. DARWIN $\square$ has been developed to supplement the current safe-life approach for low-cycle fatigue design of titanium disks/rotors in commercial aircraft gas turbine engines. DARWIND integrates finite element stress analysis, fracture mechanics analysis, nondestructive inspection simulation, and probabilistic analysis to assess the risk of rotor failure. It computes the probability-of-fracture (POF) as a function of flight cycles, considering random defect occurrence and location, random inspection schedules, and several other random variables. Both Monte Carlo and importance sampling methods are available. In addition, a user-friendly 
graphical user interface (GUI) is available to handle the otherwise difficult task of setting up the problem for analysis and viewing the results.

DARWIN consists of two entities: a computational code for performing the numerical calculations and a pre/post processing code, i.e., a Graphical User Interface (GUI) for data preparation and results visualization, see Figure 1.

Three fundamental requirements require a static permanent file be developed, 1) transferring results from the computational code to the GUI for postprocessing, 2) storaging critical data needed to be reused by the computational code, e.g., restarting, and 3 ) creating a permanent archive of the analysis.

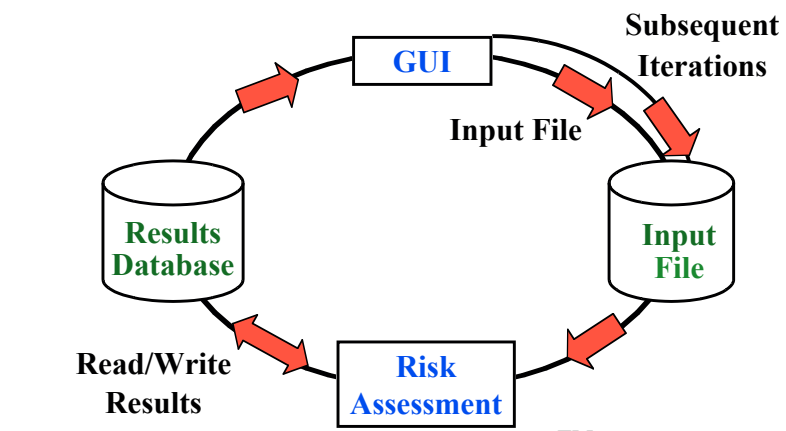

Figure 1 Interaction of the DARWIN ${ }^{\mathrm{TM}}$ components

A "zone-based" system reliability methodology is used in DARWIN ${ }^{\mathrm{TM}}$ to compute the probability-of-fracture of a disk as a function of flight cycles $[5,6]$. This methodology accounts for:

( the probability of having an anomaly in the disk,

(]) the possibility that a hard alpha anomaly developed during the titanium melt process could be in any location of the disk,

(] the initial size distribution of the anomaly,

4 randomness in the time of inspection time, probability of detection, finite element stresses and fracture mechanics analysis,

(1) the probability-of-fracture if an anomaly exists,

( the probability of detecting an anomaly and removing a disk before the disk has fractured.

A zone is a grouping of material such that all subregions in the zone have a generally uniform stress state and the same fatigue crack growth properties, inspection schedules, probability of detection curves, and anomaly distribution. In other words, the POF computed for any sub-region of material of the zone will be the same; thus, the subregions are grouped into a zone. A finite element mesh and stress results are used as the framework for the zone discretization. An example of a zoned impeller model (31 zones) superimposed on an axisymmetric finite element stress result is shown in Figure 2.

DARWIN $^{\mathrm{TM}}$ uses an iterative zone refinement procedure for risk assessment of gas turbine rotor disks to ensure that the discretization yields a sufficiently accurate answer[6]. During the iterative process, selective zones are subdivided and reanalyzed. However, many of the zones will not be modified and their results will not change. Therefore, the risk assessment code retrieves the results of the unmodified zones from the database and combines these results with results from the new zones. This can result in considerable time savings.

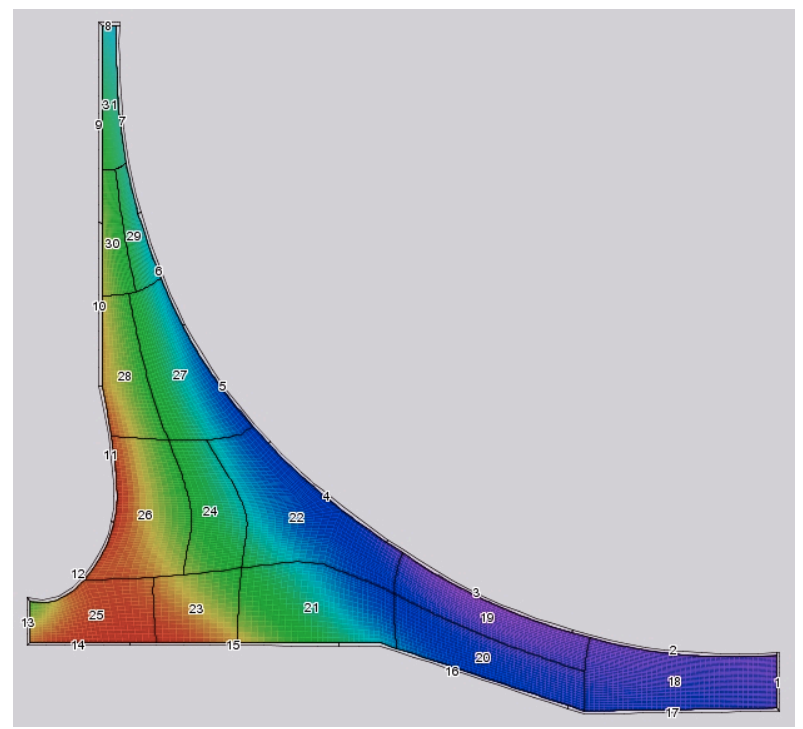

Figure 2 Zoned Impeller Model Superimposed on Axisymmetric Finite Element Stress Results

Typical outputs from DARWIN ${ }^{\mathrm{TM}}$ are the probabilityof-fracture per flight, and the risk contribution factors,. The risk contribution factors indicate the relative POF among the regions of the disk, see Figure $3 \mathrm{a}$ ) and b), respectively. 


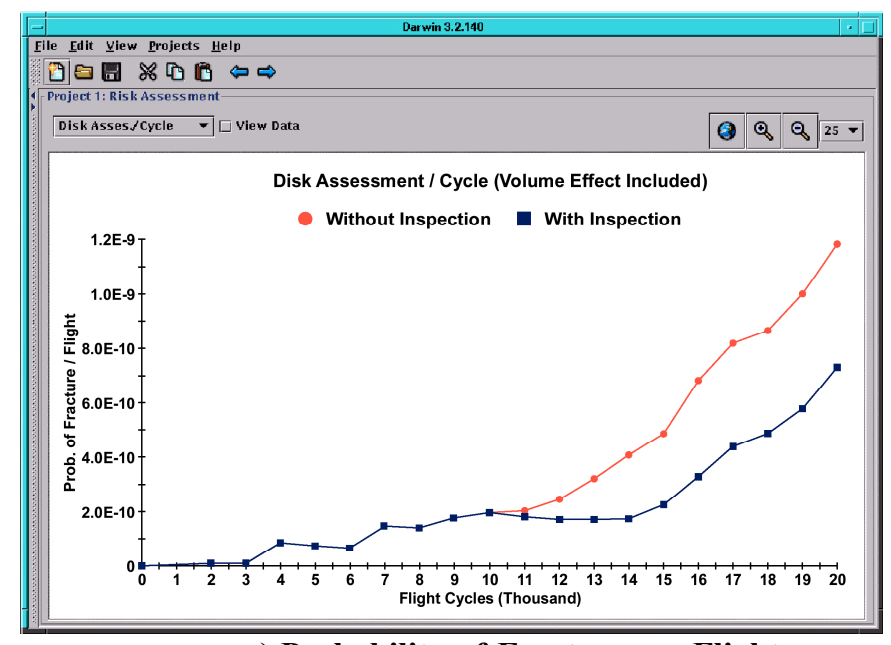

a) Probability-of-Fracture per Flight

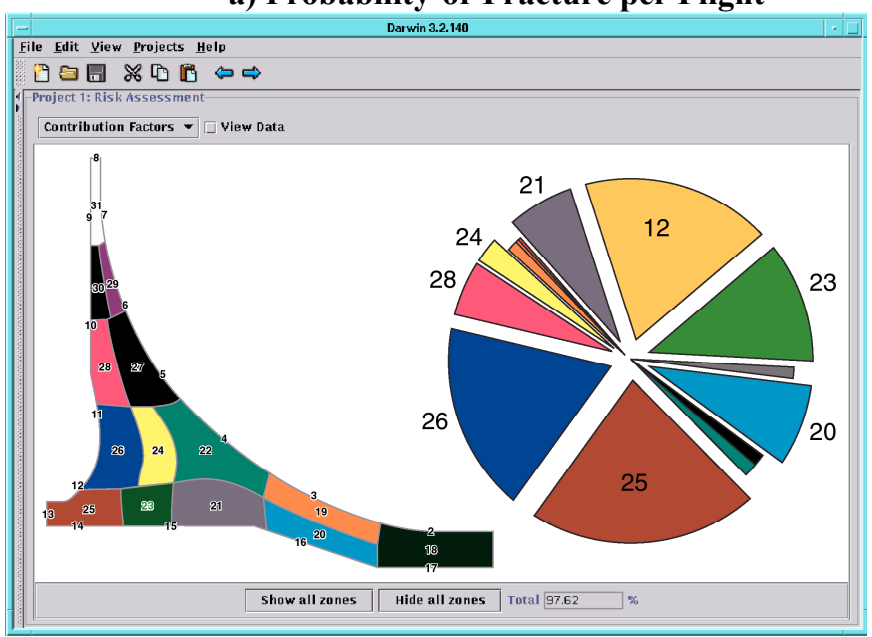

b) Risk Contribution Factors

Figure 3 DARWIN ${ }^{\mathrm{TM}}$ Outputs: a) Probability-ofFracture per Flight, b) Risk Contribution Factors

\section{Database Content}

The database contains the following information:

口 The input file data, such that the GUI can reconstruct the input file from the database.

Q All information in the text-based output file, such that the output file can be reconstructed from the database.

( All information for all zones, such that the zone information can be retrieved, combined with other new zone results, and used to compute the rotor disk probability-of-fracture.

The XML language allows the user to develop element definitions that reflect their application. Here we define element and attribute names relevant to a zone-based probabilistic analysis. The elements are nested to reflect the relationships between data. For example, Figure 4 shows the highest-level element is darwin with two subordinate elements input and output. The input element (with subordinate elements) contains all the information in the input file to the risk assessment code. The output element contains the results. The input element is expanded to reveal its subordinate elements. Some of these elements also have sub-elements, for example, the stress Input element is expanded in full to reveal sub-elements missionset $->$ mission $->$ loadCase.

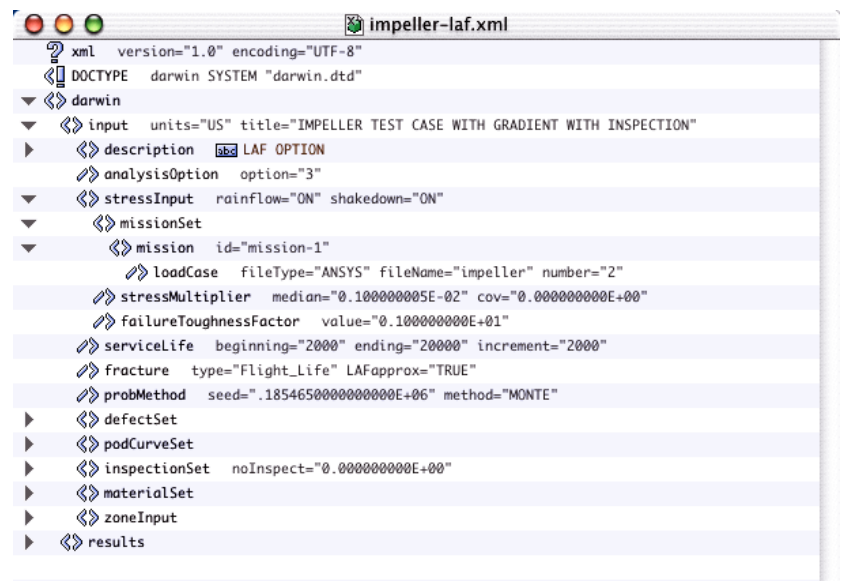

Figure 4 Top Level Database Elements

The results are subdivided by zone at the highest level since each zone is an independent analysis. Within a zone, there are three major sections: stressprocessing, avsn, risk, see Figure 5. The stressprocessing element contains the stress data for the fracture mechanics analysis, e.g., stress pairs normal to the crack plane. The avsn section contains the results of a deterministic analysis of a small flaw placed within the life limiting section of the zone. The risk section contains the results of a probabilistic analysis assuming a flaw exists in the zone. The probability of a flaw existing in the zone is considered when the zone results are combined to compute the total probability-of-fracture of the disk, i.e., unconditional POF. 


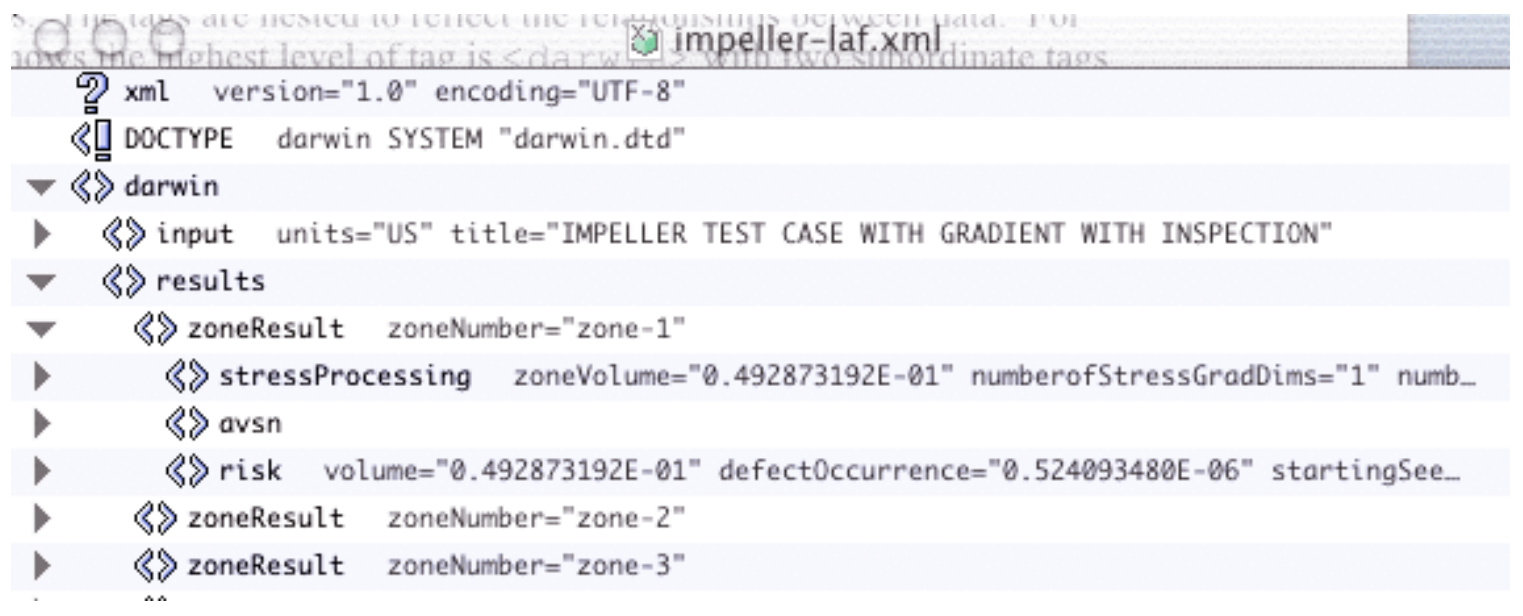

Figure 5 Results layout in the database

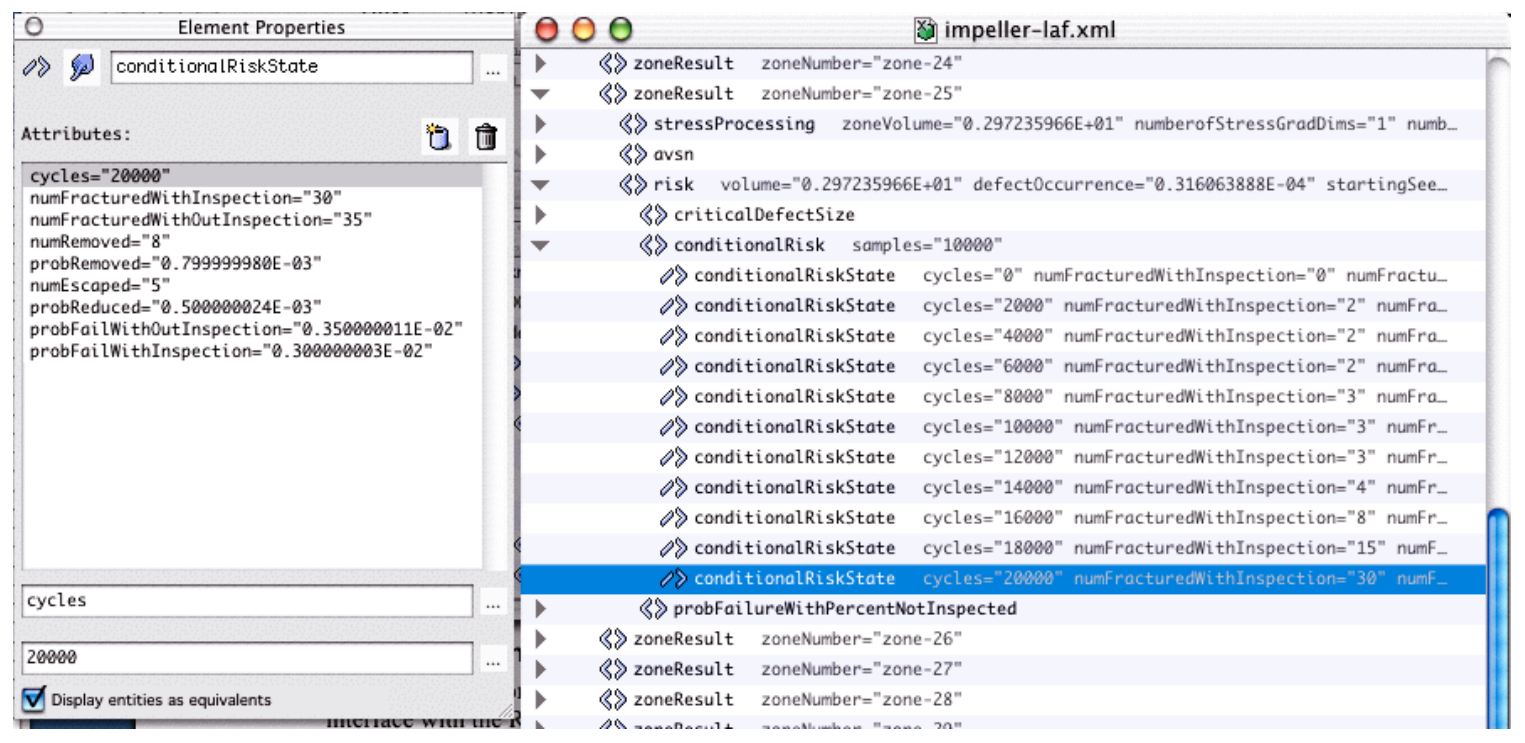

Figure 6 Conditional Probability-of-Fracture

The risk results within a zone are subdivided into three categories: criticalDefectsize - the size of a defect that will just cause failure within a specified number of cycles (all other variables assumed deterministic), conditionalRisk - the conditional probability-of-fracture assuming a defect exists in the $\mathrm{z} \quad \mathrm{O} \mathrm{n}$ e ,

a $\mathrm{n} \quad \mathrm{d}$ probFailureWithPercentNotInspected the probability-of-fracture considering inspection of the disks and removal if a flaw is detected - excluding a specified percentage of disks that are never inspected. Figure 6 shows the conditional risk results for zone 25 for every 2000 flight cycles, see Figure 2. The window on the left of Figure 6 shows the detail results at 20,000 flight cycles. The conditional probability-of-fracture without inspection (conditioned on a defect being present) is 0.0035 and the probability-of-fracture with inspection is 0.0030 .
The probability-of-fracture result for the entire disk is obtained by combining the conditional probability-offracture from the zones with the probability of a defect existing within each zone. Figure 7 shows the unconditional risk of fracture at 20,000 flight cycles for the disk. The probability-of-fracture without inspection is $5.18 \mathrm{E}-6$ and the probability-of-fracture with inspection is $4.24 \mathrm{E}-7$.

The probability-of-fracture of the disk per flight cycle without inspection is $2.59 \mathrm{E}-11$, with lower and upper $95 \%$ confidence bounds of $2.23 \mathrm{E}-11$ and $2.96 \mathrm{E}-11$, respectively, see Figure 8 . The probability-of-fracture of the disk per flight cycle with inspection is $2.12 \mathrm{E}-11$ with lower and upper $95 \%$ confidence bounds of $1.80 \mathrm{E}-$ 11 and $2.45 \mathrm{E}-11$, respectively. 


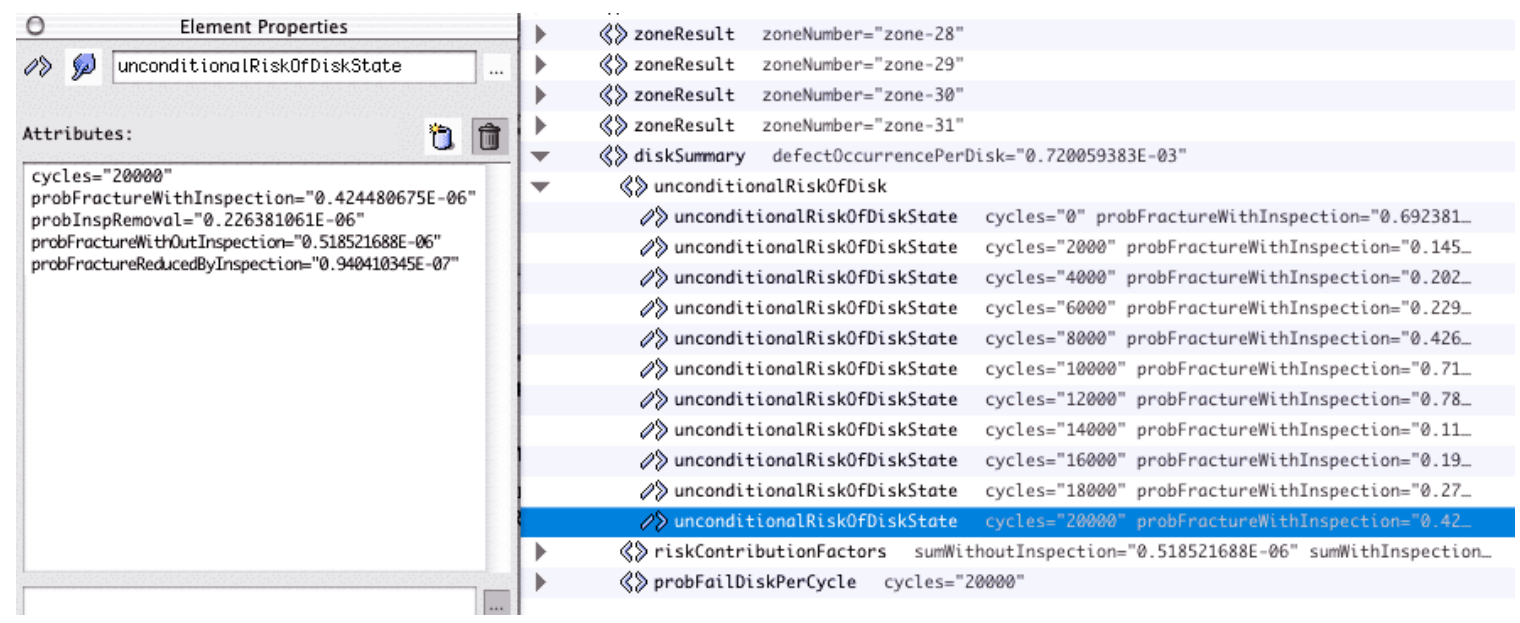

Figure 7 Unconditional Probability-of-Fracture

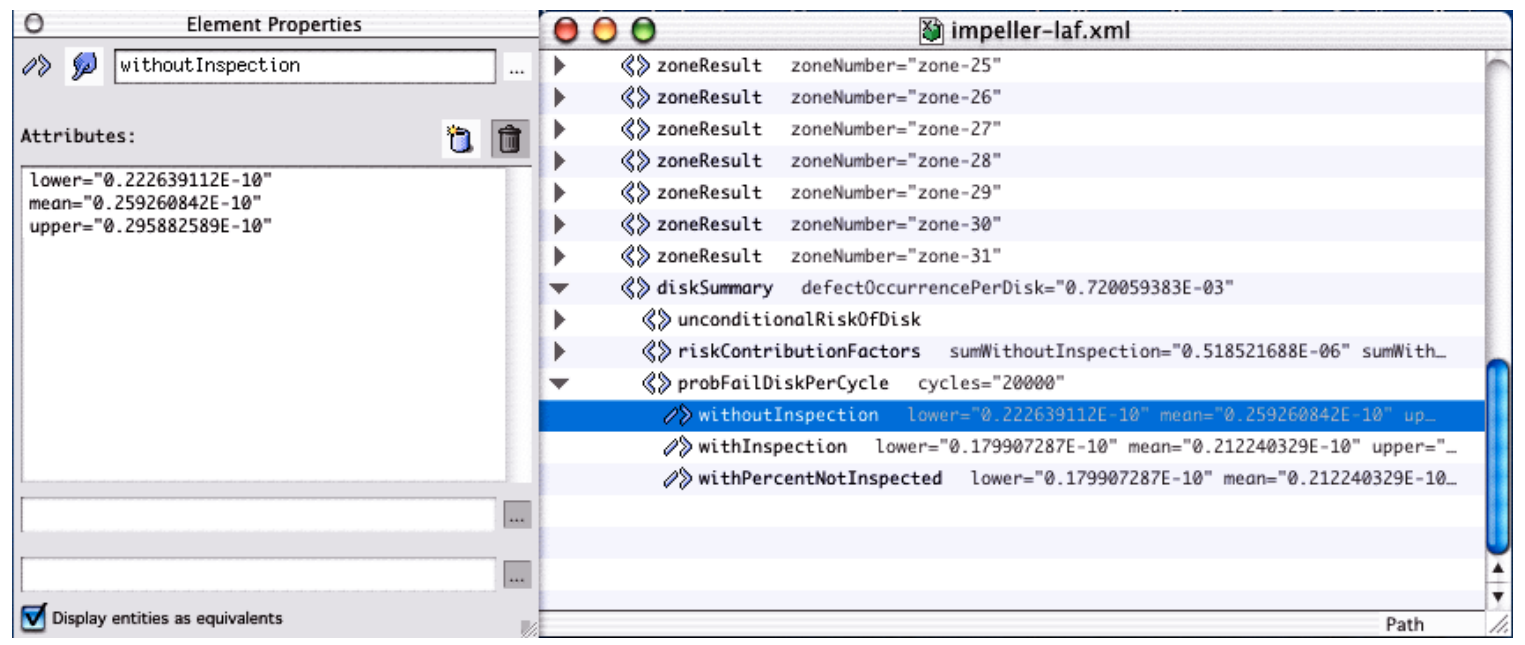

Figure 8 Probability-of-Fracture per Flight

$\underline{\text { Database }} \underline{\text { Validation }}=\underline{\text { using }} \underline{\text { the }} \underline{\text { Document }} \underline{\text { Type }}$ $\underline{\text { Definition }}$

An XML-based file format has the ability to be syntactically self-validating, meaning that the XML data file provides metadata describing rules that the data file must obey [7]. This metadata is in a form known as the Document Type Definition (DTD) ${ }^{1}$. The DTD formally declares the accepted element and attribute names, as well as structure of the document and constraints on values. The DTD allows an application to check that an XML file is syntactically accurate before processing it semantically. A well designed DTD is critical to developing an XML file format that both meets current application requirements

\footnotetext{
${ }^{1}$ There is a standard called XML Schema in development to replace DTD. See http://www.w3.org/XML/Schema
}

and provides means of extensibility for future needs. If an XML document fails the DTD check, then the data file is in some way deficient and must be corrected.

The DTD is most valuable to the programmer during new development. As the database is modified during the development cycle, for example to add new features, the database can be very easily validated against the DTD and any errors in writing the database can be caught during the development cycle where it is most effective to correct. Therefore, the approach when modifying the database is to first update the DTD to specify the new database structure, then modify the code to generate the new database format, run example problems and validate the new databases against the DTD.

Figure 9 below shows a small subset of the DTD for DARWIN $^{\mathrm{TM}}$. Entities are used to define the permissible values. For example, entity boolean can 
be any of (TRUE|FALSE |ON |OFF|YES|NO) but no other. unitType can be either US or METRIC. These entities are referenced throughout the

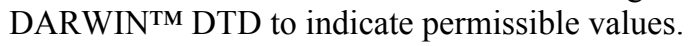

The top level element darwin has two subordinate elements input and results with results being optional, and two attributes "version" and "guiversion", i.e., the versions of the risk assessment code and graphical interface used for the analysis, which are both strings. The input element has thirteen potential subordinate elements as shown. resultsDatabase, gradientset, inspectionset and the combined pair (podCurveset, inspectionset) are optional. The input element has three attributes analysis, units and title. The analysis attribute must be of type analysistype, i.e. surface or inherent, the units attribute must be of type unitType, i.e., US or METRIC, and title attribute must be of type string, i.e., any character string. In this manner, the DTD defines the entire set of allowable elements and attributes. Thus, given the DTD and an XML file, a validating parser can verify that the file contains acceptable data.

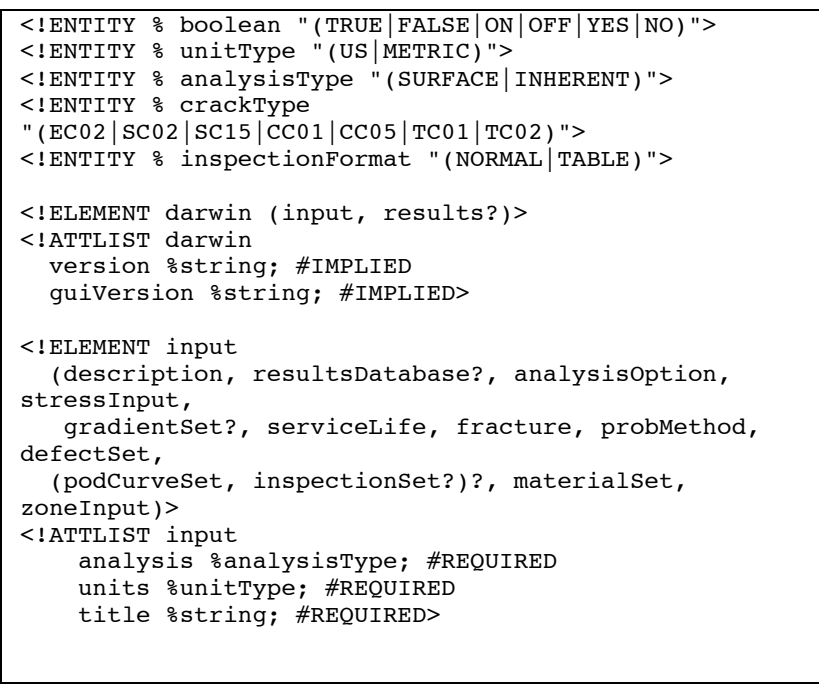

\section{Figure 9 DTD subset for DARWIN ${ }^{\text {TM }}$}

There are several convenient publicly available tools to validate the database. The publicly available $r \times p$ software accepts on input an XML file and a DTD[8]. If errors are located, rxp prints the offending line number and the error.

\section{Summarv and Conclusions}

In this work, we explore the applicability of using XML as a database file format for a probabilistic fracture mechanics program. The primary use of the database is to store and retrieve data during computations and to transfer the computational results to the graphical user interface for post-processing.

Validation of a database, i.e., correct placement of elements and attributes is a substantial aid during code modifications. Publicly available software programs ensure validation upon given a DTD and a database file. This allows the developers to easily detect and then correct errors in the computer programming.

An ancillary benefit to using an XML format is that the database can be easily modified using stylesheets. One use for this feature is to generate reports in HTML format. We provide a mechanism whereby the user can designate what information is wanted in a report summary and an HTML report generated.

A related benefit from the use of stylesheets is that modifications to the database file can be accomplished easily. This is especially useful when upgrading a database format to a newer version. A stylesheet can be developed such that the users can upgrade older databases to a newer format.

In summary, using an XML file format database as a means to transfer data between components of a computer program and archive the results is very practical for most programs and has significant advantages compared to the traditional approach of developing application specific customized database formats. We expect the use of XML to become a standard tool for the scientific programmer.

\section{Acknowledgments}

This work was supported by the Federal Aviation Administration (FAA) under Grant 99-G-016. The authors wish to thank Bruce Fenton and Joe Wilson (FAA Technical Center) and Tim Mouzakis (FAA Engine and Propeller Directorate) for their continued support and encouragement. other team members, Gerald Leverant, Craig McClung and Yi-Der Lee of Southwest Research Institute. The ongoing contributions of the Southwest Research Institute team members, Gerald Leverant, Craig McClung, Yi-Der Lee, and Graham Chell, and of the Industry Steering Committee (Darryl Lehmann, Pratt \& Whitney; Jon Tschopp, General Electric; Ahsan Jameel, Honeywell; Jon Dubke, Rolls-Royce) are also gratefully acknowledged.

\section{$\underline{\text { References }}$}

1. XML-W3 - http://www.w3.org/XML/ 
2. Leverant, G.R., McClung, R.C., Millwater, H.R., and Enright, M.P., 2003, "A New Tool for Design and Certification of Aircraft Turbine Rotors", Journal of Engineering for Gas Turbines and Power, ASME (accepted).

3. M.P. Enright, Y.-D. Lee, R.C. McClung, L. Huyse, G.R. Leverant, H.R. Millwater, S.H.K. Fitch, "Probabilistic Surface Damage Tolerance Assessment of Aircraft Turbine Rotors," ASME International Gas Turbine Conference (IGTI), June 16-19, 2003, Atlanta, GA, Paper no. GT200338731

4. H.R. Millwater, S.H.K. Fitch, Y.-T. Wu, D. Riha, M.P. Enright, G. Leverant, R.C. McClung, C. Kuhlman, G. Chell, Y.-D. Lee, "A Probabilistically-based Damage Tolerance Analysis Computer Program for Hard Alpha Anomalies in Titanium Rotors," ASME International Gas Turbine Conference (IGTI), ASME Paper No. 2000-GT-0421, Munich, Germany, May 8-11, 2000

5. Wu, Y.-T., Enright, M.P., and Millwater, H., "Probabilistic Methods for Design Assessment of Reliability with Inspection", AIAA Journal, V40, No. 5, pp 937-946, 2002

6. H. Millwater, M. Enright, "A Convergent Probabilistic Technique for Risk Assessment of Gas Turbine Disks Subject to Metallurgical Defects," AIAA Structures, Structural Dynamics, and Materials Conference, AIAA Paper No. 20021382, Denver, CO, April Atlanta, GA, April 22-25, 2002

7. Goldfarb, C.F., Prescod, P., The XML Handbook, 1998, Prentice-Hall, Inc.

8. $\mathrm{r} \times \mathrm{p} \quad \mathrm{S}$ o $\mathrm{f} \mathrm{t}$ w $\mathrm{a} \mathrm{r}$, http://www.cogsci.ed.ac.uk/ richard/rxp.html 